\title{
STRICT RADICAL CLASSES OF COMMUTATIVE RINGS
}

\author{
N. R. MCCONNELL
}

(Communicated by Maurice Auslander)

\begin{abstract}
We classify strongly hereditary strict radical classes for commutative rings and give some results for supernilpotent strict radical classes and strict radical classes contained in the nil radical.
\end{abstract}

In this paper (with a few exceptions) we restrict our domain to the variety of commutative rings. The technique of restricting study to a smaller class of rings in order to get simplifications has been used by Widiger and Wiegandt [12] and van Leeuwen [6], who studied the class of hereditarily artinian rings, and by Gardner [5], who studied the class of regular rings with artinian primitive images.

Sands [8] studied the relationship between various radical properties (such as strictness, stability, etc.) for associative rings. He displays 23 different combinations of these properties that are satisfied by at least one radical class of associative rings. On considering commutative rings only, we find the story much simpler. These 23 combinations reduce to six, mainly because any hereditary radical class of commutative rings is left and right hereditary, and every radical class of commutative rings is left and right stable (and left and right strong). The six are as follows: satisfying no properties, hereditary, strongly hereditary, strict, hereditary strict, and strongly hereditary strict; thus study of strict radical classes looms large in radical theory of commutative rings. The first section is inspired by Stewart's characterisation of strongly hereditary strict radical classes of associative rings.

It should be noted here that all the results of Snider [9] on the lattice of radicals of associative rings carry over in the obvious way to commutative rings. We denote " $S$ is a subring of $A$ " by $S<A$. For radical theoretic terminology, the reader is referred to [13].

\section{STRONGLY HEREDITARY STRICT RADICALS}

It has been shown by Stewart [11] that the only strongly hereditary strict radical classes in the class of associative rings (besides $\{0\}$ and the class of all rings) are the classes $\mathscr{T}_{P}$, for $P$ a set of primes, consisting of rings in which every element has order a product of powers of primes from $P$. Thus every $\mathscr{T}_{P}$ is contained in $\mathscr{T}$, the radical class of torsion rings.

Received by the editors March 12, 1991 and, in revised form, August 26, 1991.

1991 Mathematics Subject Classification. Primary 16A21. 
It is also known that the nil radical class $\mathscr{N}$ is strongly hereditary and strict in the class of commutative rings. The question then arises: Are there any strongly hereditary strict radical classes of commutative rings other than meets and joins of the nil radical and the radicals $\mathscr{T}_{P}$ ? Note that here a meet of radical classes (denoted by $\Lambda$ ) is their intersection and the join of radical classes (denoted by $V$ ) is the radical class defined by the intersection of their semisimple classes. We will need the following four results.

Lemma 1.1. Let $\mathscr{R}$ be a hereditary strict radical class. If $A^{0}$ denotes the ring with trivial multiplication on the additive group of $A$, then $A^{0} \in \mathscr{R}$ whenever $A \in \mathscr{R}$.

Proof. Suppose $A \in \mathscr{R}$. Then by [11, Proposition 3.1(ii)], $A[X] \in \mathscr{R}$ (as $\mathscr{R}$ is strict) and the ideal $A X$ is in $\mathscr{R}$, since $\mathscr{R}$ is hereditary. Then $A^{0} \cong$ $A X / A X^{2} \in \mathscr{R}$.

Proposition 1.2. Let $A$ be a torsionfree commutative semiprime ring. Then $A$ is a subdirect product of torsionfree prime rings.

Proof. For any prime ideal $P$ of $A$, let $\widehat{P}=\left\{a \in A \mid n a \in P\right.$ for some $\left.n \in \mathbb{Z}^{+}\right\}$. For $a, b \in \widehat{P}, n a, m b \in P$, and $r \in A$, we have $m n(a-b)=m(n a)-n(m b) \in$ $P$ and $m(r a)=r(m a) \in P$, so $\widehat{P} \triangleleft A$. Also, for $c, d \in A, c d \in \widehat{P}$, and $k(c d) \in P$ we have $(k c) d \in P$, so $k c \in P$ or $d \in P$ (as $P$ is a prime ideal). But then either $c \in \widehat{P}$ or $d \in \widehat{P}$, so $\widehat{P}$ is a prime ideal of $A$.

Now, for $0 \neq t \in A$, let $S=\left\{m t^{n} \mid m, n \in \mathbb{Z}^{+}\right\}$. Then $S$ is multiplicatively closed, and as $A$ is torsionfree, $0 \notin S$. Thus we can find an ideal $M_{t}$ maximal with respect to disjointness from $S ; M_{t}$ is prime, so $\widehat{M}_{t}$ is prime also. Since $k t \in S$ for any $k \in \mathbb{Z}^{+}$and $S$ is disjoint from $M_{t}, k t$ can never be an element of $M_{t}$ and so $t \notin \widehat{M}_{t}$.

Finally, if $i a \in \widehat{M}_{t}$ for some positive integer $i, a \in A$, then there is a positive integer $j$ with $j(i a) \in M_{t}$, that is, $(j i) a \in M_{t}$ and so $a \in \widehat{M}_{t}$. Hence $A / \widehat{M}_{t}$ is torsionfree and prime. Since also $t \notin \widehat{M}_{t}$ for any nonzero $t$, we have $\bigcap\left\{\widehat{M}_{t} \mid 0 \neq t \in A\right\}=0$, so that $A$ is a subdirect product of the prime, torsionfree rings $A / \widehat{M}_{t}$.

Proposition 1.3. Let $R$ be a semiprime, commutative, torsionfree ring. Either $R$ has a subring isomorphic to $m \mathbb{Z}$ for some $m \in \mathbb{Z}^{+}$or the subring generated by any nonzero element of $R$ is isomorphic to the free ring on one generator.

Proof. If $R$ has an identity then the subring generated by it will be isomorphic to $\mathbb{Z}$, so we assume $R$ has no identity.

First suppose that there is an $m \in \mathbb{Z}^{+}, a \in R, a \neq 0$, such that $a^{2}=m a$. Define a function $f: m \mathbb{Z} \rightarrow\langle a\rangle$, where $\langle a\rangle=\{n a \mid n \in \mathbb{Z}\}$, the subring of $R$ generated by $a$, by $f(m n)=n a$. Then $f(m n)+f(m k)=n a+k a=$ $f(m n+m k)$ and $f(m n) \cdot f(m k)=n a k a-n k m a=f(m n k m)=f(m n \cdot m k)$, so $f$ is a homomorphism. Since $R$ has characteristic $0, f(m n)=0$ if and only if $m n=0$, so $f$ is injective. Since $f$ is clearly surjective, $f$ is an isomorphism. Thus $m \mathbb{Z}$ is isomorphic to a subring of $R$.

Second, suppose that for every nonzero $a \in R$, there is no $m \in \mathbb{Z}^{+}$with $a^{2}=m a$. Now $X \mathbb{Z}[X]=\left\{n_{1} X+\cdots+n_{m} X^{m} \mid n_{i} \in \mathbb{Z}\right\}$ is the free commutative 
ring on one generator. Define $h: X \mathbb{Z}[X] \rightarrow\langle a\rangle$ by

$$
h\left(n_{1} X+\cdots+n_{m} X^{m}\right)=n_{1} a+\cdots+n_{m} a^{m} .
$$

$h$ is clearly a surjective homomorphism with kernel $K=\left\{n_{1} X+\cdots+n_{m} X^{m} \mid\right.$ $\left.n_{1} a+\cdots+n_{m} a^{m}=0\right\}$. Suppose that $K \neq 0$. Let $n_{k} X^{k}+\cdots+n_{m} X^{m} \in K$ (where $k$ is the smallest power of $X, m$ the largest) with $n_{k} n_{m} \neq 0$, so $n_{k} a^{k}+a^{k}\left(n_{k+1} a+\cdots+n_{m} a^{m-k}\right)=0$. Then

$$
n_{k} a^{k}=-\left(n_{k+1} a+\cdots+n_{m} a^{m-k}\right) a^{k},
$$

so there is an element $b$ of $\langle a\rangle$ with $n_{k} a^{k}=b a^{k}$, and $b \neq 0$ since $R$ is torsionfree. But we now have $\left(n_{k}\right)^{k} a^{k}=b^{k} a^{k} \neq 0$ (since $A$ is torsionfree and $a$ is not nilpotent), so $\left(b^{k}\right)^{2}=\left(n_{k}\right)^{k} b^{k}$ (since $b^{k}$ is a polynomial in $a$ with smallest power of $a$ at least $\left.a^{k}\right)$. This contradicts our assumption above that there was no element $c \neq 0$ of $A$ with $c^{2}=j c$ for some integer $j$. Thus $h$ is an isomorphism, and so $\langle a\rangle \cong X \mathbb{Z}[X]$ for any nonzero $a \in R$.

Proposition 1.4. The lattice of strongly hereditary radicals of associative rings is a complete sublattice of the lattice of all radicals.

Proof. It is clear that the meet of any collection of strongly hereditary radicals is strongly hereditary, so we need only show that arbitrary joins of strongly hereditary radicals are strongly hereditary. Let $\left\{\mathscr{R}_{i}\right\}$ be a collection of strongly hereditary radicals, and let $\mathscr{R}$ be their join. Let $R$ be a ring in $\mathscr{R}$. Then by [9, Lemma 2] there exists a chain $\left\{I_{\lambda}\right\}$ of ideals of $R$ (where the $\lambda$ are ordinals) with $I_{0}=0, I_{\lambda+1} / I_{\lambda} \in \mathscr{R}_{i}$ for some $i, I_{\sigma}=\bigcup\left\{I_{\lambda} \mid \lambda<\sigma\right\}$ for limit ordinals $\sigma$, and $\bigcup_{\lambda} I_{\lambda}=R$. Let $S<R$. Then $\left\{S \cap I_{\lambda}\right\}$ is a chain of ideals of $S$, and we need only verify that $\left(S \cap I_{\lambda+1}\right) /\left(S \cap I_{\lambda}\right) \in \mathscr{R}_{i}$ for some $i$. Now

$$
\left(S \cap I_{\lambda+1}\right) /\left(S \cap I_{\lambda}\right) \cong\left(S \cap I_{\lambda+1}+I_{\lambda}\right) / I_{\lambda} \subseteq I_{\lambda+1} / I_{\lambda} \in \mathscr{R}_{i}
$$

for some $i$; so since all the $\mathscr{R}_{i}$ are strongly hereditary, we have $S \in \bigvee \mathscr{R}_{i}$ as required.

The question of what the strongly hereditary strict radical classes of commutative rings look like can be divided into seven cases (where throughout $\mathscr{R}$ is a strongly hereditary strict radical class):

(i) $\mathscr{N} \vee \mathscr{T} \subseteq \mathscr{R}$. Here we can in fact show that either $\mathscr{R}$ is the class of all rings or $\mathscr{R} \subseteq \mathscr{N} \vee \mathscr{T}$ as follows. Suppose that $\mathscr{R} \nsubseteq \mathscr{N} \vee \mathscr{T}$, and let $A$ be in $\mathscr{R} \backslash \mathscr{N} \vee T$. Then $0 \neq A /(\mathscr{N} \vee \mathscr{T})(A)$ is torsionfree and semiprime, so by Proposition 1.3 it has a subring of the form $m \mathbb{Z}(m \neq 0)$ or $X \mathbb{Z}[X]$. As $\mathscr{R}$ is strongly hereditary, this subring is in $\mathscr{R}$. If $m \mathbb{Z} \in \mathscr{R}$, then $\mathbb{Q} \in \mathscr{R}$ by strictness, and hence $\mathbb{Z} \in \mathscr{R}$. But then $\mathbb{Z}[X] \in \mathscr{R}$ by [11, Proposition 3.1(ii)], so $X \mathbb{Z}[X] \in \mathscr{R}$. Thus $X \mathbb{Z}[X] \in \mathscr{R}$ in any case, so $\mathscr{R}$ contains all one-generator rings and, therefore, all rings.

(ii) $\mathscr{R} \subseteq \mathscr{N} \wedge \mathscr{T}$. Armendariz [1] showed that for associative rings, the only nonzero hereditary subradicals of the prime radical, are the classes $\beta \wedge$ $\mathscr{T}_{P}$, where $\beta$ denotes the prime radical. His proof works equally well for commutative rings, so (since $\beta=\mathscr{N}$ for commutative rings) the only nonzero possibilities here are the $\mathscr{N} \wedge \mathscr{T}_{P}$.

(iii) $\mathscr{R} \subseteq \mathscr{T}, \mathscr{R} \nsubseteq \mathscr{N}$. Suppose that there is an $A \in \mathscr{R}$ such that $A \notin \mathscr{N}$. Then $A / \mathscr{N}(A)$ is a nil-semisimple $\mathscr{R}$-ring, so it is a subdirect product of prime 
$\mathscr{R}$-rings. Let $R$ be one such ring. Then $R \in \mathscr{R} \subseteq \mathscr{T}$, so $R$ has characteristic $p$ for some prime $p$ and is thus in $\mathscr{T}_{p}$. Then the field $Q(R)$ of quotients of $R$ is in $\mathscr{R}$, and so is $\mathbb{Z}_{p}$ (as $Q(R)$ has a subfield isomorphic to $\mathbb{Z}_{p}$ ). Thus $\mathbb{Z}_{p}[X] \in \mathscr{R}, X \mathbb{Z}_{p}[X] \in \mathscr{R}$, and all 1-generated rings of characteristic $p$ are in $\mathscr{R}$. It follows that all rings of characteristic $p$ are in $\mathscr{R}$.

In particular, $\mathbb{Z}_{p}^{0} \in \mathscr{R}$. Let $A \in \mathscr{N} \wedge \mathscr{T}_{p}$. If $a \in A$ has order $p$ and $a^{n}=0 \neq a^{n-1}$, then the subring generated by $a^{n-1}$ is isomorphic to $\mathbb{Z}_{p}^{0}$. Since $\mathscr{R}$ is strict, there is an ideal of $A$ in $\mathscr{R}$; thus every nonzero homomorphic image of every ring of $\mathscr{T}_{p} \wedge \mathscr{N}$ has a nonzero $\mathscr{R}$-ideal and is thus in $\mathscr{R}$, so $\mathscr{N} \wedge \mathscr{T}_{p} \subseteq \mathscr{R}$. Hence for any $p$-ring $A, \mathscr{N}(A) \in \mathscr{R}$ and $A / \mathscr{N}(A)$ has characteristic $p$ (as a semiprime $p$-ring), so $A / \mathscr{N}(A) \in \mathscr{R}$ and $A \in \mathscr{R}$. We thus have $\mathscr{T}_{p} \subseteq \mathscr{R}$ if and only if $\mathscr{R}$ contains one prime $p$-ring. Let $P$ be the set of primes $p$ for which $\mathscr{R}$ contains a prime $p$-ring. Then $\mathscr{T}_{P} \subseteq \mathscr{R}$ and no other nil-semisimple $\mathscr{T}$-rings are contained in $\mathscr{R}$. Now, by (ii), there is a set $Q$ of primes for which $\mathscr{R} \wedge \mathscr{N}=\mathscr{T}_{Q} \wedge \mathscr{N}$. Then $\left(\mathscr{T}_{Q} \wedge \mathscr{N}\right) \vee \mathscr{T}_{P} \subseteq \mathscr{R}$. If $R$ is any $\mathscr{R}$-ring and $R \notin \mathscr{N}$, then $R / \mathscr{N}(R)$ is a nil-semisimple $\mathscr{T}$-ring, so as above $R / \mathscr{N}(R) \in \mathscr{T}_{P}$. Also $\mathscr{N}(R) \in \mathscr{T}_{Q} \wedge \mathscr{N}$, so $R \in \mathscr{T}_{P} \vee\left(\mathscr{T}_{Q} \wedge \mathscr{N}\right)$. Thus $\mathscr{R}=\mathscr{T}_{P} \vee\left(\mathscr{T}_{Q} \wedge \mathscr{N}\right)$.

(iv) $\mathscr{R} \subseteq \mathscr{N}, \mathscr{R} \nsubseteq \mathscr{T}$. Let $A$ be an $\mathscr{R}$-ring with $A \notin \mathscr{T}$. Then $R=$ $A / \mathscr{T}(A)$ is a nonzero $\mathscr{T}$-semisimple ring and $R \in \mathscr{N}$ as $R \in \mathscr{R} \subseteq \mathscr{N}$. Let $a \in R$. Then $a^{n}=0$ for some $n \in \mathbb{Z}^{+}$. Let $n$ be minimal such that $a^{n}=0$, let $m=[(n+1) / 2]$, and let $b=a^{m}$. Then $b^{2}=0$ and $m b \neq 0$ for all $m \in \mathbb{Z}^{+}$, so $\langle b\rangle$ is isomorphic to $\mathbb{Z}^{0}$. Thus $\mathbb{Z}^{0} \in \mathscr{R}$, so $\mathscr{N} \subseteq \mathscr{R}$, as $\mathbb{Z}^{0}$ generates $\mathscr{N}$; that is, $\mathscr{R}=\mathscr{N}$.

(v) $\mathscr{T} \subseteq \mathscr{R}, \mathscr{N} \nsubseteq \mathscr{R} . \mathscr{T} \subseteq \mathscr{R} \subsetneq \mathscr{N} \vee \mathscr{T}$. If $R \in \mathscr{R}$ with $R \notin \mathscr{T}$, then $R^{0} \in \mathscr{R}$ by Lemma 1 , and as in (iv) $\mathscr{N} \subseteq \mathscr{R}$-a contradiction. Thus $\mathscr{R}=\mathscr{T}$.

(vi) $\mathscr{N} \subseteq \mathscr{R}, \mathscr{T} \nsubseteq \mathscr{R} . \mathscr{N} \subseteq \mathscr{R} \varsubsetneqq N \vee \mathscr{T}$. As in (iii), there is a largest set $P$ of primes such that $\mathscr{T}_{P} \subseteq \mathscr{R}$. If $R \in \mathscr{R}$, then by [9, Lemma 2] there is a chain $\left\{I_{\lambda}\right\}$ of ideals of $R$ with $I_{\lambda+1} / I_{\lambda}$ in either $\mathscr{N}$ or $\mathscr{T}$ for every $\lambda$, and with $\bigcup_{\lambda} I_{\lambda}=R$. As in (iii), those $I_{\lambda+1} / I_{\lambda}$ not in $\mathscr{N}$ must be in $\mathscr{T}_{P}$, so $R \in \mathscr{T}_{P} \vee \mathscr{N}$, that is, $\mathscr{R}=\mathscr{T}_{P} \vee \mathscr{N}$.

(vii) $\mathscr{N}, \mathscr{T}$, and $\mathscr{R}$ are independent. Here we can show that either $\mathscr{R} \subseteq \mathscr{T}$ or $\mathscr{N} \subseteq \mathscr{R}$, so that this case does not occur.

If there is a ring $A \in \mathscr{R} \backslash \mathscr{T}$ then $0 \neq A / \mathscr{T}(A) \in \mathscr{R}$. If $A / \mathscr{T}(A)$ is not nil, then it has a torsionfree semiprime image $B \in \mathscr{R}$. But then $0 \neq B \in$ $\mathscr{R} \backslash \mathscr{T} \vee \mathscr{N}$, so $\mathscr{R}$ is the class of all rings. But if $A / \mathscr{T}(A) \in \mathscr{N}$ then $\mathscr{N} \subseteq \mathscr{R}$ (as in (iv)), so when $\mathscr{R}$ is not the class of all rings either $\mathscr{R} \subseteq \mathscr{T}$ or $\mathscr{N} \subseteq \mathscr{R}$.

We thus have

Theorem 1.5. A nonzero, proper radical class of commutative rings is strongly hereditary and strict if and only if $\mathscr{R}$ can be obtained by taking meets and joins of the radical classes $\mathscr{N}$ and $\mathscr{T}_{P}$ for various sets $P$ of primes.

It can thus be seen that the strongly hereditary strict radicals form a complete sublattice of the lattice of all radicals of commutative rings (cf. [9]).

\section{SUPERNILPOTENT STRICT RADICALS}

As noted in (ii) of the previous section, the hereditary subradicals of $\mathscr{N}$ are known, and they are all strongly hereditary and strict. At the other end of the spectrum, however, Gardner [4] showed that for any subidempotent, hereditary 
radical $\mathscr{R}$, the polynomial ring $A[X] \notin \mathscr{R}$ for any ring $A \neq 0$. If $\mathscr{R}$ is also strict, it follows that $\mathscr{R}=\{0\}$. Thus there are no nonzero hereditary strict subidempotent radicals (and note that this applies for associative rings also). Throughout this section we will assume that $\mathscr{R}$ is a strict, supernilpotent radical class of commutative rings.

If $\mathscr{R}$ properly contains the nil radical class $\mathscr{N}$, then there is a ring $R \in \mathscr{R}$ with $\mathscr{N}(R)=0$. Then $R$ is a subdirect product of $\mathscr{R}$-radical prime rings $\left\{A_{\lambda} \mid \lambda \in \Lambda\right\}$. If $Q(A)$ denotes the field of quotients of a prime ring $A$, then, for each $\lambda, A_{\lambda} \subseteq \mathscr{R}\left(Q\left(A_{\lambda}\right)\right)$ by the strictness of $\mathscr{R}$, and thus $Q\left(A_{\lambda}\right) \in \mathscr{R}$. Thus $\mathscr{R}$ contains fields.

Now let $\mathscr{F}$ be the class of all fields. If $\mathscr{F} \cap \mathscr{R}=\varnothing$ then $\mathscr{R}=\mathscr{N}$ as above, so $\mathscr{F} \cap \mathscr{R}=\varnothing$ if and only if $\mathscr{R}=\mathscr{N}$. On the other hand, $\mathscr{F} \cap \mathscr{R}=\mathscr{F}$ does not mean that $\mathscr{R}$ is the class of all rings, since the upper radical determined by $\mathbb{Z}$ and its subrings (= ideals) is strict (and hereditary) and contains no fields. In fact, we can say more than this.

Let $\mathscr{B}$ denote the class of all rings such that no subring is isomorphic to $X \mathbb{Z}[X]$, the free ring on one generator. Then $\mathscr{B}$ is a radical class (Stewart [10]). Clearly $\mathscr{B}$ is strongly hereditary. By [7, Theorem 2.5], the smallest strict radical class containing $\mathscr{B}$ (which we denote $\mathscr{B}_{<}$) has semisimple class

$$
\mathscr{S P B}<=\{A \mid S<A \Rightarrow \mathscr{B}(S)=0\} \text {. }
$$

Now let $\mathscr{P}$ denote the class of rings such that every 1 -generated subring is isomorphic to $X \mathbb{Z}[X]$, and suppose $A \in \mathscr{P}$. Let $S<A$. Then $\langle x\rangle \cong X \mathbb{Z}[X]$ for all $x \in S$, so there is no $I \triangleleft S$ with $\langle x\rangle \not X \mathbb{Z}[X]$ for all $x \in I$. Thus $\mathscr{B}(S)=0$ for all $S<A$, so $A \in \mathscr{S} \mathscr{B}<$.

Conversely, suppose $A \in \mathscr{S P}_{<} ;$then $\mathscr{B}(\langle x\rangle)=0$ for all $x \in A$. Thus every ideal of $\langle x\rangle$ has an element that generates a free subring; in particular, there is an $a \in\langle x\rangle$ with $\langle a\rangle \cong X \mathbb{Z}[X]$. Suppose $n_{1} x+\cdots+n_{m} x^{m}=0$ for integers $n_{1}, \ldots, n_{m}, n_{m} \neq 0$, and let $G\langle a, b, \ldots\rangle$ denote the additive abelian group generated by $a, b, \ldots$ (see [3] for abelian group-theoretic terminology). Then we have $0 \neq n_{m} x^{m}=-n_{1} x+\cdots+n_{m-1} x^{m-1} \in G\left\langle x, x^{2}, \ldots, x^{m-1}\right\rangle$. Suppose that for some $k>m$ there is a $j \in \mathbb{Z} \backslash\{0\}$ such that $j x^{k}=j_{1} x+\cdots+j_{m-1} x^{m-1}$ for integers $j_{1}, \ldots, j_{m-1}$. Then

$$
\begin{aligned}
n_{m} j x^{k+1} & =n_{m}\left(j_{1} x+\cdots+j_{m-1} x^{m-1}\right) x \\
& =\left(n_{m} j_{1} x^{2}+\cdots+n_{m} j_{m-2} x^{m-1}\right)+j_{m-1} n_{m} x^{m} \\
& =\left(n_{m} j_{1} x^{2}+\cdots+n_{m} j_{m-2} x^{m-1}\right)-j_{m-1}\left(n_{1} x+\cdots+n_{m-1} x^{m-1}\right) \\
& \in G\left\langle x, \ldots, x^{m-1}\right\rangle .
\end{aligned}
$$

It follows by induction that $x^{m}, x^{m+1}, \ldots$ have finite order modulo $G\left\langle x, \ldots, x^{m-1}\right\rangle$; hence $G\left\langle x, x^{2}, \ldots\right\rangle / G\left\langle x, \ldots, x^{m-1}\right\rangle$ is a torsion group, and thus $G\left\langle x, x^{2}, \ldots\right\rangle$ has finite torsionfree rank; so $G\left\langle x, x^{2}, \ldots\right\rangle$ has no free (abelian) subgroup of infinite rank.

Now $G\left\langle x, x^{2}, \ldots\right\rangle=\langle x\rangle^{+}$and $\langle a\rangle$ is a free subring of $\langle x\rangle$, so $\langle a\rangle^{+} \subseteq$ $G\left\langle x, x^{2}, \ldots\right\rangle$-a contradiction, as $\langle a\rangle$ has infinite rank. Thus $n_{1} x+\cdots+$ $n_{m} x^{m}=0$ only if all the $n_{i}$ are zero, so $\langle x\rangle \cong X \mathbb{Z}[X]$ provided $x \neq 0$. Thus $\mathscr{P}=\mathscr{S} \mathscr{B}<$.

Now, by [7, Theorem 2.5] again, the radical class corresponding to $\mathscr{S O B}_{<}$is $\mathscr{B}_{<}=\{A \mid A / I \neq 0 \Rightarrow 0 \neq S<A / I$ for some $S \in \mathscr{B}\}$; that is, the set of rings 
such that every homomorphic image has a nonzero subring in which no element generates a free ring. We see that $\mathscr{B} \neq \mathscr{B}_{<}$as, for example, $\mathbb{Z}[X] \in \mathscr{B}<$ (with the subring generated by 1 in any homomorphic image never isomorphic to $X \mathbb{Z}[X])$ but $\mathbb{Z}[X] \notin \mathscr{B}$ (as $X$ generates $X \mathbb{Z}[X])$.

The argument can be repeated working modulo a prime $p$, so that if we define $\mathscr{B}_{p}=\left\{A \mid\right.$ no 1 -generated subring of $A$ is isomorphic to $\left.X \mathbb{Z}_{p}[X]\right\}$, we have $\mathscr{S O S}_{p_{<}}=\left\{A \mid\right.$ every subring of $A$ has an element that generates $\left.X \mathbb{Z}_{p}[X]\right\}$, and the corresponding radical class is $\mathscr{B}_{p_{<}}=\{A \mid$ every nonzero homomorphic image of $A$ has a nonzero subring in $\left.\mathscr{B}_{p}\right\}$.

Now let $\mathscr{D}$ be the radical class consisting of all rings with divisible additive groups. We can now prove

Proposition 2.1. Let $\mathscr{R}$ be a strict supernilpotent radical class of commutative rings.

(i) $\mathscr{Q} \in \mathscr{R}$ if and only if $\mathscr{D} \cap \mathscr{B}_{<} \subseteq \mathscr{R}$;

(ii) $\mathbb{Z}_{p} \in \mathscr{R}$ if and only if $\mathscr{T}_{p} \cap \mathscr{B}_{p_{<}} \subseteq \mathscr{R}$.

Proof. (i) Let $\mathscr{R}$ be as stated, and suppose that $\mathscr{D} \cap \mathscr{B}<\subseteq \mathscr{R}$. Then $\mathbb{Q}$ has no subring isomorphic to $X \mathbb{Z}[X]$, so (as $\mathbb{Q}$ is simple), $\mathbb{Q} \in \mathscr{B}_{<}$, and thus $\mathbb{Q} \in \mathscr{R}$.

Conversely, let $\mathbb{Q} \in \mathscr{R}$ and $R \in \mathscr{D} \cap \mathscr{B}<$. Without loss of generality, we can assume that $R$ is semiprime and hence torsionfree (as torsion divisible rings are nilpotent). Suppose $R$ has no nonzero idempotents. If $a \in R$ satisfies $a^{2}=m a$ for some $m \in \mathbb{Z}^{+}$, let $b \in R$ with $a=m b$ (possible since $R$ is divisible). Then

$$
m^{2} b^{2}=(m b)^{2}=a^{2}=m a=m^{2} b,
$$

so by torsionfreeness $b=b^{2}$ and $a=b=0$. Thus no $m \mathbb{Z}$ is isomorphic to any subring of $R$, so by Proposition 1.3 every 1 -generated subring of $R$ is

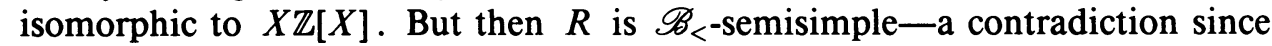
$R \in \mathscr{B}_{<}$. Thus $R$ has a nonzero idempotent $e$. By divisibility and torsionfreeness, there are unique elements $e_{1}=e, e_{2}, \ldots$ of $R$ with $n e_{n}=e$ for each $n \in \mathbb{Z}^{+}$. Let $E=\left\{m \cdot e_{n} \cdot e \mid m \in \mathbb{Z}, n \in \mathbb{Z}^{+}\right\}$. Then we have

$$
n q\left(m \cdot e_{n} \cdot e-p \cdot e_{q} \cdot e\right)=q m \cdot e^{2}-p n \cdot e^{2}=(q m-p n) e,
$$

so $m \cdot e_{n} \cdot e-p \cdot e_{q} \cdot e=n q\left(m \cdot e_{n} \cdot e-p \cdot e_{q} \cdot e\right) e_{n q}=(q m-p n) e_{n q} \cdot e \in E$. Also

$$
m \cdot e_{n} \cdot e \cdot p \cdot e_{q} \cdot e=m p \cdot e_{n} \cdot e_{q} \cdot e=m p \cdot e_{n q} \cdot e \in E
$$

so $E$ is a subring of $R$. Defining $f: \mathbb{Q} \rightarrow E$ by $f(m / n)=m \cdot e_{n} \cdot e$, we see that $f$ is an isomorphism, so $R$ has a subring isomorphic to $\mathbb{Q}$, and thus, by the strictness of $\mathscr{R}$, a nonzero $\mathscr{R}$-ideal.

Now let $\bar{R}$ be a nonzero homomorphic image of $R$. Then $\bar{R} \in \mathscr{D} \cap \mathscr{B}_{<}$ also. If $\mathscr{N}(\bar{R}) \neq 0$ then $\mathscr{N}(\bar{R})$ is a nonzero $\mathscr{R}$-ideal of $\bar{R}$. Similarly, if $\mathscr{T}(\bar{R}) \neq 0$ then $\mathscr{T}(\bar{R}) \in \mathscr{N}$, and so $\mathscr{T}(\bar{R})$ is a nonzero $\mathscr{R}$-ideal of $\bar{R}$. Finally, if $\mathscr{N}(\bar{R})=\mathscr{T}(\bar{R})=0$ then the arguments of the previous paragraph apply to $\bar{R}$, and so $\bar{R}$ has a nonzero $\mathscr{R}$-ideal. Thus every nonzero homomorphic image of $R$ has a nonzero $\mathscr{R}$-ideal, so $R \in \mathscr{R}$.

(ii) Clearly $\mathbb{Z}_{p} \in \mathscr{B}_{p<}$, so $\mathbb{Z}_{p} \in \mathscr{R}$ if $\mathscr{B}_{p<} \cap \mathscr{T}_{p} \subseteq \mathscr{R}$. Conversely, suppose $\mathbb{Z}_{p} \in \mathscr{R}$, and let $A \in \mathscr{B}_{p<} \cap \mathscr{T}_{p}$. We can assume that $A$ is semiprime, so $A$ is a subdirect product of prime rings of characteristic $p$ and hence has characteristic $p$. Let $a \in A$ with $\langle a\rangle \not X X \mathbb{Z}_{p}[X]$, so there is a set $\left\{n_{k}, \ldots, n_{m}\right\}$ 
of nonnegative integers less than $p$ with $n_{k} n_{m} \neq 0$ such that $n_{k} a^{k}+\cdots+$ $n_{m} a^{m}=0$. Suppose $m$ is minimal; then

$$
n_{k} a^{k}=a^{k}\left(-n_{k+1} a-\cdots-n_{m} a^{m-k}\right) \neq 0
$$

(as $\left.0<n_{k}<p\right)$, so letting $b=\left(-n_{k+1} a-\cdots-n_{m} a^{m-k}\right)$ we have $\left(n_{k}\right)^{k} a^{k}=$ $b^{k} a^{k} \neq 0$ (since $\left(n_{k}\right)^{k}$ is prime to $p$ and $n_{k}$ is not nilpotent). Thus (as in the proof of Proposition 1.3) $\left(b^{k}\right)^{2}=\left(n_{k}\right)^{k} b^{k}$. Let $0<j<p$ with $j \equiv$ $\left(n_{k}\right)^{k}(\bmod p)$, and write $b^{k}=c$. Since $j$ and $p$ are relatively prime, there are integers $s$ and $t$ with $s j+t p=1$. Then

$$
(s c)^{2}=s^{2} c^{2}=s^{2} j c=(1-t p) s c=s c,
$$

so the subring generated by $s c$ is isomorphic to $\mathbb{Z}_{p}$. Thus $A$ has a subring isomorphic to $\mathbb{Z}_{p}$ and, by the strictness of $\mathscr{R}$, an $\mathscr{R}$-ideal. If $\bar{A}$ is a nonzero homomorphic image of $A$, then either $\mathscr{N}(\bar{A})$ is a nonzero $\mathscr{R}$-ideal of $A$ or the arguments above apply to $\bar{A}$; in either case, any nonzero homomorphic of $A$ has an $\mathscr{R}$-ideal, so $A \in \mathscr{R}$.

Since $\mathbb{Q} \in \mathscr{R}$ means that every field of characteristic 0 is in $\mathscr{R}$ and $\mathbb{Z}_{p} \in \mathscr{R}$ means that every field of characteristic $p$ is in $\mathscr{R}$, we have

Corollary 2.2. $\mathscr{H}=\bigvee_{p}\left(\mathscr{T}_{p} \wedge \mathscr{B}_{p<}\right) \vee\left(\mathscr{D} \wedge \mathscr{B}_{<}\right) \vee \mathscr{N}$ is the smallest supernilpotent strict radical class of commutative rings that contains all fields.

\section{STRICT RADICALS CONTAINED IN THE NIL RADICAL}

As seen in $\S 1$, the torsion radical $\mathscr{T}$ and the nil radical $\mathscr{N}$ are both strongly hereditary and strict. The divisible radical $\mathscr{D}$ is also strict (since the divisible radical of a ring $R$ is the maximal divisible subgroup of the additive group of $R)$, but is not hereditary. Another radical class that is strict but not hereditary is the class of all idempotent rings (that is, rings $R$ satisfying $R^{2}=R$ ). We will focus our attention in this section on the interplay between these four radical classes and consider those strict radicals contained in $\mathscr{N}$.

Consider the algebra $Z(F, K)$ over a field $F$ generated by the set $\left\{x_{\alpha} \mid 0<\right.$ $\alpha<1, \alpha \in K\}$ where $K$ is a field, with $\mathbb{Q} \subseteq K \subseteq \mathbb{R}$ and multiplication defined by

$$
x_{\alpha} x_{\beta}= \begin{cases}x_{\alpha+\beta}, & \alpha+\beta<1, \\ 0, & \alpha+\beta \geq 1,\end{cases}
$$

and extended linearly to other elements (with $K=\mathbb{Q}$, this is known as the Zassenhaus algebra over $F)$. In fact we can define $Z(R, K)$ for any commutative ring $R$, but for the moment we will only use $Z(F, K)$. For any $F$ and $K, Z(F, K)$ is idempotent, since, for any $\alpha, x_{\alpha}=\left(x_{\alpha / 2}\right)^{2}$. Also $Z(F, K)$ is nil, since for any $\alpha$ there is an $n \in \mathbb{Z}^{+}$with $n \alpha>1$, and thus $x_{\alpha}^{n}=0$. Thus $Z(F, K) \in \mathscr{I} \wedge \mathscr{N}$ for any $F$ and $K$. It is shown in [2] that $Z(F, \mathbb{R})$ is unequivocal where $F$ is $\mathbb{Q}$ or a prime field; this proof works equally well for any $F$ and $K$, and we see also that $Z(F, K)$ is divisible and torsionfree or a reduced $p$-ring exactly when $F$ is. Furthermore, $Z(F, K) \subseteq Z(G, L)$ if $F \subseteq G$ and $K \subseteq L$.

Now suppose that $\mathscr{M}$ is a strongly hereditary class of rings with $Z(F, K) \in$ and $Z(G, L) \notin \mathscr{M}$ where $Z(F, K) \subseteq Z(G, L)$ (provided $Z(F, K) \not$ 
$Z(G, L)$, which is certainly true if $F \neq G$ or $|K| \neq|L|)$. Let $\mathscr{R}$ denote the upper radical with respect to $\mathscr{M}$. Then $\mathscr{R}=\{A \mid A$ has no nonzero homomorphic image in $\mathscr{M}\}$ and is strict. By slightly adapting the reasoning in [2], we see that every homomorphic image of $Z(G, L)$ has the form

$$
Z(G, L) / I=\left\{c x_{\alpha}+\sum a_{\beta} x_{\beta} \mid 0<\beta<\alpha, c \in G / H\right\}
$$

where $G / H$ is some additive quotient group of $G, x_{\sigma} x_{\tau}=x_{\sigma+\tau}$ if $\sigma+\tau<\alpha$ and 0 , if $\sigma+\tau \geq \alpha, a x_{\sigma} b x_{\tau}=(a b+H) x_{\alpha}$ when $\sigma+\tau=\alpha$. If we consider the subring $S$ of $Z(G, L) / I$ defined as for $Z(G, L) / I$ but with $c=0$ always, then the function $f: Z(G, L) \rightarrow S$ defined by $f\left(x_{\sigma}\right)=x_{\alpha \sigma}$ is easily seen to be an isomorphism. Thus every homomorphic image of $Z(G, L)$ has a subring isomorphic to $Z(G, L) \notin \mathscr{M}$ and thus no homomorphic image of $Z(G, L)$ can be in $\mathscr{M}$ since $\mathscr{M}$ is strongly hereditary. Thus $Z(G, L) \in \mathscr{R}$.

This means that for any fields $F, G, K$, and $L$ as above there is at least one strict radical class $\mathscr{R}$ with $Z(G, L) \in \mathscr{R}$ and $Z(F, K) \notin \mathscr{R}$, so the lattice of strict radical classes contained in $\mathscr{I} \wedge \mathscr{N}$ is at least as complicated as the set $\{Z(F, K) \mid F, K$ fields, $\mathbb{Q} \subseteq K \subseteq \mathbb{R}\}$ partially ordered by inclusion. We will thus turn to those strict radicals $\mathscr{R}$ with $\mathscr{R} \subseteq \mathscr{N}$ and $\mathscr{R} \wedge \mathscr{I}=\{0\}$; here things turn out to be much simpler.

Theorem 3.1. Let $\mathscr{R}$ be a strict radical class of commutative rings contained in $\mathscr{N}$ with $\mathscr{R} \wedge \mathscr{I}=\{0\}$. Then $\mathscr{R}=\mathscr{T}_{N} \wedge \mathscr{D}$ for some set $N$ of primes.

Proof. Let $\mathscr{R}$ be as in the statement of the theorem. Then $\mathscr{R} \neq \mathcal{N}$, as there are nil idempotent rings. Let $A$ be a nil-ring with $\mathscr{R}(A)=0$. Then every subring $S$ of $A$ is a nil-ring with $\mathscr{R}(S)=0$. Let $a \in A, a^{n}=0 \neq a^{n-1}$. Then $\langle a\rangle=\left\{m_{1} a+\cdots+m_{n-1} a^{n-1} \mid m_{i} \in \mathbb{Z}\right\}$ and $\langle a\rangle^{n}=0$, so $\langle a\rangle^{n-1}=\left\{m a^{n-1} \mid m \in\right.$ $\mathbb{Z}\} \cong \mathbb{Z}^{0}$ or $\mathbb{Z}_{k}^{0}$ (depending on whether $a^{n-1}$ has infinite order or order $k$ additively). We know $\mathbb{Z}^{0} \notin \mathscr{R}$ (as otherwise $\mathscr{R}=\mathscr{N}$ ). If $\mathbb{Z}_{k}^{0} \notin \mathscr{R}$ then $\mathbb{Z}_{p}^{0} \notin \mathscr{R}$ for some $p \mid k$. Let $P$ be the set of primes for which $\mathbb{Z}_{p}^{0} \notin \mathscr{R}$. Then $q \in Q$, the set of primes not in $P$, means that $\mathbb{Z}_{q}^{0} \in \mathbb{R}$. Thus $\mathscr{T}_{Q} \wedge \mathscr{N} \subseteq \mathscr{R}$ (as in $\S 1$ (iii)). But then $Z\left(\mathbb{Z}_{q}, K\right) \in \mathscr{R}$ for any $K, q \in Q$ and $Z\left(\mathbb{Z}_{q}, K\right)$ is idempotent, so $P$ must be the set of all primes.

Let $A \in \mathscr{R}, p \in P$, and suppose $A \neq p A$. Then $0 \neq A / p A \in \mathscr{R}$, so $(A / p A)^{+}$is a direct sum of copies of $\mathbb{Z}_{p}^{+}$. Also $(A / p A)^{2} \neq A / p A$ and $(A / p A)^{2}=\left(A^{2}+p A\right) / p A \cong A^{2} /\left(A^{2} \cap p A\right)$, so

$$
0 \neq(A / p A) /(A / p A)^{2}=(A / p A) /\left(\left(A^{2}+p A\right) / p A\right) \cong A /\left(A^{2}+p A\right) \in \mathscr{R} .
$$

$\left(A /\left(A^{2}+p A\right)\right)^{+}$is a homomorphic image of $A / p A^{+}$, and thus a direct sum of copies of $\mathbb{Z}_{p}^{+}$. Also $\left(a+\left(A^{2}+p A\right)\right)\left(b+\left(A^{2}+p A\right)\right)=0$ for all $a, b \in A$, so $A /\left(A^{2}+p A\right)$ is a zero-ring and thus is isomorphic to a direct sum of copies of $\mathbb{Z}_{p}^{0}$ and hence has a homomorphic image isomorphic to $\mathbb{Z}_{p}^{0}$-a contradiction, as $\mathbb{Z}_{p}^{0} \notin \mathbb{R}$. Thus $p A=A$ for all $p \in P$ and $A \in \mathscr{R}$, and so all $\mathscr{R}$-rings are divisible.

Since there are idempotent rings in $\mathscr{D} \wedge \mathscr{N}$ (for example, $Z(F, K)$ for any $K$ and any $F$ of characteristic 0$)$, we must have $\mathbb{Q}^{0} \notin \mathscr{R}$. Now any $A \in \mathscr{R}$ is divisible, so $A$ is additively a direct sum of full rational and quasicyclic subgroups and $(A / \mathscr{T}(A))^{+}$is a direct sum of copies of $\mathbb{Q}$. Suppose that $A \neq \mathscr{T}(A)$. Then $(A / \mathscr{T}(A)) /(A / \mathscr{T}(A))^{2} \neq 0$. Let $A / \mathscr{T}(A)=R$. Then for a 
typical element $\sum a_{i} b_{i}$ of $R^{2}$ and for any $n \in \mathbb{Z}^{+}$we can find elements $a_{1}^{\prime}$ of $R$ with $n a_{i}^{\prime}=a_{i}$ for every $i$ by divisibility. Thus $n r-\sum a_{i} b_{i}=n\left(r-\sum a_{i}^{\prime} b_{i}\right) \neq 0$ unless $r \in R^{2}$ by torsionfreeness of $R$, so $R / R^{2}$ is torsionfree. Hence $A$ maps homomorphically onto a zero-ring on a direct sum of copies of $\mathbb{Q}$, so $A$ maps onto $\mathbb{Q}^{0}$-a contradiction, so we see that $A=\mathscr{T}(A)$, and so $\mathscr{R} \subset \mathscr{T} \wedge \mathscr{D}$. As shown in [8], the only radicals of associative rings contained in $\mathscr{T} \wedge \mathscr{D}$ are the $\mathscr{T}_{N} \wedge \mathscr{D}$ for sets $N$ of primes; thus $\mathscr{R}=\mathscr{T}_{N} \wedge \mathscr{D}$ for some such set, since all the $\mathscr{T} \wedge \mathscr{D}$ rings are commutative, and each $\mathscr{T}_{N} \wedge \mathscr{D}$ is strict.

\section{REFERENCES}

1. E. P. Armendariz, Hereditary subradicals of the lower Baer radical, Publ. Math. (Debrecen) 15 (1968), 91-93.

2. N. Divinsky, Unequivocal rings, Canad. J. Math. 27 (1975), 679-690.

3. L. Fuchs, Infinite abelian groups, Vols. I, II, Academic Press, New York, 1970, 1973.

4. B. J. Gardner, A note on radicals and polynomial rings, Math. Scand. 31 (1972), 83-88.

5. __ Radical classes of regular rings with Artinian primitive images, Pacific J. Math. 99 (1982), 337-349.

6. L. C. A. van Leeuwen, Complements of radicals in the class of hereditarily Artinian rings, Acta Sci. Math. 39 (1977), 313-318.

7. N. R. McConnell, Hereditary and strict domains for radical classes of associative rings, Bull. Austral. Math. Soc. 41 (1990), 255-269.

8. A. D. Sands, On dependence and independence among radical properties, Rings, Modules and Radicals (Proceedings of the Hobart Conference 1987), Pitman Research Notes in Math., vol. 204, Longman Sci. Tech., Essex, 1989.

9. R. L. Snider, Lattices of radicals, Pacific J. Math. 40 (1972), 207-220.

10. P. N. Stewart, Strongly hereditary radical classes, J. London Math. Soc. (2) 4 (1972), 499509.

11. __ Strict radical classes of associative rings, Proc. Amer. Math. Soc. 39 (1973), 273-278.

12. A. Widiger and R. Wiegandt, Theory of radicals for hereditarily Artinian rings, Acta Sci. Math. 39 (1977), 303-312.

13. R. Wiegandt, Radical and semisimple classes of rings, Queen's Papers in Pure and Appl. Math., vol. 37, Queen's Univ., Kingston, Ontario, 1974.

Department of Mathematics and Computing, University College of Central QueEnsland, RockHampton, QueEnsland, 4702 Australia

E-mail address: mcconneln@topaz.ucq.edu.au 